\title{
Syringocystadenoma papilliferum in an unusual location beyond the head and neck region: A case report and review of literature
}

\begin{abstract}
A case of syringocystadenoma papilliferum with multiple papulonodules in a linear fashion located in an unusual location of the right lower abdomen is presented. The presence of a large tumor at the inferior pole raised the suspicion of malignant transformation and the presence of discharge from the lesions raised the possibility of necrosis. However, histopathological examination showed the classical features of syringocystadenoma papilliferum without malignant transformation or tumor necrosis. The patient refused to undergo surgical excision of the nodules and subsequently was lost to follow-up. This case illustrates the atypical location of a rare disease and adds to the differential diagnosis of linear verrucous lesions on the abdomen. Review of all the cases with syringocystadenoma papilliferum outside the head and neck region in the English literature showed only one case of syringocystadenoma papilliferum arising on the abdomen; our patient is the second reported case with the unique feature of linear arrangement of lesions.
\end{abstract}

Item Type: Article

Keyword: Dermatopathology; Syringocystadenoma papilliferum 